\title{
CORRECTION
}

\section{Correction to: The child and adolescent psychiatry: study of training in Europe (CAP-STATE)}

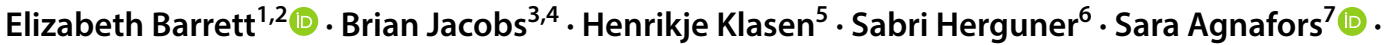

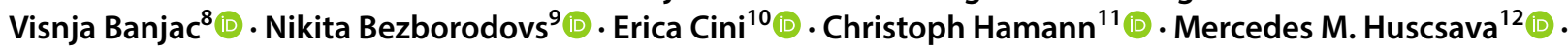 \\ Maya Kostadinova ${ }^{13,14}$. Yuliia Kramar $^{15} \cdot$ Vanja Mandic Maravic ${ }^{16} \cdot$ Jane McGrath $^{17}$ (1) $\cdot$ Silvia Molteni ${ }^{18}$. \\ Maria Goretti Moron-Nozaleda ${ }^{19}$ [ . Susanne Mudra ${ }^{20}$ (1) . Gordana Nikolova ${ }^{21} \cdot$ Kallistheni Pantelidou Vorkas $^{22}$. \\ Ana Teresa Prata ${ }^{23}$ (D) Alexis Revet ${ }^{24}$. Judeson Royle Joseph ${ }^{25}$ (D) Reelika Serbak ${ }^{26} \cdot$ Aran Tomac $^{27}$. \\ Helena Van den Steene ${ }^{28}\left[\right.$. Georgios Xylouris ${ }^{29}$. Anna Zielinska ${ }^{30}$. Johannes Hebebrand ${ }^{31}$
}

Published online: 19 January 2020

(c) Springer-Verlag GmbH Germany, part of Springer Nature 2020

\section{Correction to: European Child \& Adolescent Psychiatry} https://doi.org/10.1007/s00787-019-01416-3

In the original article, the thirteenth author name has incorrectly published as Vanda Mandic Maravic. The correct name is Vanja Mandic Maravic.

The original article has been corrected.

The original article can be found online at https://doi.org/10.1007/ s00787-019-01416-3.

Elizabeth Barrett

Elizabeth.barrett@ucd.ie

Extended author information available on the last page of the article 


\section{Affiliations}

\section{Elizabeth Barrett ${ }^{1,2}$ D $\cdot$ Brian Jacobs $^{3,4} \cdot$ Henrikje Klasen $^{5} \cdot$ Sabri Herguner $^{6} \cdot$ Sara Agnafors $^{7}$ (D) . Visnja Banjac $^{8} \mathbb{D} \cdot$ Nikita Bezborodovs $^{9} \mathbb{D} \cdot$ Erica Cini $^{10} \mathbb{D} \cdot$ Christoph Hamann $^{11} \mathbb{D} \cdot$ Mercedes M. Huscsava $^{12}$. $^{1}$ Maya Kostadinova ${ }^{13,14}$ (D) . Yuliia Kramar ${ }^{15}$. Vanja Mandic Maravic ${ }^{16}$. Jane McGrath ${ }^{17}$ (D) Silvia Molteni $^{18}$. Maria Goretti Moron-Nozaleda ${ }^{19}$. Susanne Mudra ${ }^{20}$. Gordana Nikolova $^{21} \cdot$ Kallistheni Pantelidou Vorkas $^{22}$. Ana Teresa Prata ${ }^{23}$ (D) Alexis Revet ${ }^{24}(D)$ Judeson Royle Joseph ${ }^{25}$ (D) Reelika Serbak ${ }^{26} \cdot$ Aran Tomac $^{27}$. Helena Van den Steene ${ }^{28}$ (D) Georgios Xylouris ${ }^{29}$. Anna Zielinska ${ }^{30}$. Johannes Hebebrand ${ }^{31}$}

Brian Jacobs

brian.jacobs@kcl.ac.uk

Sabri Herguner

herguners@yahoo.com

Sara Agnafors

sara.agnafors@liu.se

Visnja Banjac

visnjab76@hotmail.com

Nikita Bezborodovs

nikita.bezborodovs@rsu.lv

Erica Cini

ericacini@gmail.com

Christoph Hamann

christoph.hamann@gmx.de

Mercedes M. Huscsava

mercedes.huscsava@meduniwien.ac.at

Maya Kostadinova

maia_kostadinova@abv.bg

Yuliia Kramar

voitenko.julia@gmail.com

Vanja Mandic Maravic

vanjamandic81@gmail.com

Jane McGrath

Jane.mcgrath@tcd.ie

Silvia Molteni

silvimolt@yahoo.it

Maria Goretti Moron-Nozaleda mgoretti.moron@salud.madrid.org

Susanne Mudra

s.mudra@uke.de

Gordana Nikolova

gordana.shaleva@gmail.com

Kallistheni Pantelidou Vorkas pankallistheni@cytanet.com.cy

Ana Teresa Prata

atprata5@hotmail.com

Alexis Revet

alexisrevet@gmail.com

Judeson Royle Joseph

Judeson.joseph@unn.no

Reelika Serbak

reelika.serbak@gmail.com
Anna Zielinska

ania.b.zielinska@gmail.com

1 Child and Adolescent Psychiatry, University College Dublin, Dublin, Ireland

2 Child and Adolescent Liaison Psychiatry, Children's University Hospital, Temple St., Dublin 1, Ireland

3 Child and Adolescent Psychiatry, South London and Maudsley Hospital, London, UK

4 Section of Child and Adolescent Psychiatry, European Union of Medical Specialists (UEMS-CAP), Brussels, Belgium

5 Department of Psychiatry, Leiden University Medical Centre, Leiden, Netherlands

6 Child and Adolescent Psychiatry, Private Practice, Ankara, Turkey

7 Division of Child and Adolescent Psychiatry, Department of Clinical and Experimental Medicine, Faculty of Health Sciences, Linköping University, 58185 Linköping, Sweden

8 Clinic of Psychiatry, University Clinical Center of the Republic of Srpska, Radoja Domanovica 21, Banjaluka, Bosnia and Herzegovina

9 Department of Psychiatry and Narcology, Riga Stradins University, Tvaika iela 2, Riga 1005, Latvia

10 Child and Adolescent Psychiatrist, East London Foundation Trust, East London Foundation Trust, London, UK

11 University Hospital of Child and Adolescent Psychiatry and Psychotherapy, University of Bern, Forschungsabteilung KJP UPD Bern, Bolligenstrasse 111, Haus A, 3000 Bern 60, Switzerland

12 Department of Child and Adolescent Psychiatry, Medical University of Vienna, Währinger Gürtel 18-20, 1090 Vienna, Austria

13 Child and Adolescent Psychiatrist, University Hospital "Alexandrovska", Sofia, Bulgaria

14 Present Address: DNCC CAMHS, 44 North Great George's Street, Dublin 1, Ireland

15 TMA “PSYCHIATRY”, Kirilivska str. 103, Kiev, Ukraine

16 Department for Psychotic Disorders, Institute of Mental Health, Belgrade, Serbia

17 Child and Adolescent Psychiatrist, Linn Dara Child and Adolescent Mental Health Service, Cherry Orchard Hospital, Ballyfermot, Dublin 10, Ireland 
18 Child Neuropsychiatry Unit, Department of Brain and Behavioral Sciences, University of Pavia, via Mondino 2, 27100 Pavia, Italy

19 Department of Psychiatry and Clinical Psychology, Neurodevelopment Outpatient Clinic and Day Hospital for Pre-pubertal Children, Niño Jesús Children's Hospital, Hospital Infantil Universitario Niño Jesús, av/Menéndez Pelayo 65, 28009 Madrid, Spain

20 Department of Child and Adolescent Psychiatry, Psychotherapy und Psychosomatics, University Medical Center Hamburg-Eppendorf, Martinistr. 52, W35, 20246 Hamburg, Germany

21 Department of Child and Adolescent Psychiatry, University Clinic of Psychiatry, Belgradska b.b, 1000 Skopje, Macedonia

22 Child and Adolescent Psychiatrist, President of Cypriot Society of Child and Adolescent Psychiatry, 77, Kennedy Ave, 1076 Nicosia, Cyprus

23 Child and Adolescent Psychiatry Specialty, Centro Hospitalar de Lisboa Central, Hospital Dona Estefânia, Rua Jacinta Marto, 1169-045 Lisbon, Portugal

24 Child and Adolescent Psychiatrist, Department of Child and Adolescent Psychiatry, Faculty of Medicine, Toulouse University Hospital (CHU de Toulouse), UMR1027, INSERM, University of Toulouse III, Toulouse, France
Child and Adolescent Psyciatric Department, University Hospital of North-Norway, Troms $\varnothing$, Norway

26 Child Psychiatrist, Tallinn Children`s Hospital, Tervise 28, Tallinn, Estonia

27 Child and Adolescent Psychiatry and in General (Adult) Psychiatry, CAMHS Clare, Unit 6, Quin Rd. Business Pk. Quin Rd., Ennis, Ireland

28 Collaborative Antwerp Psychiatric Research Institute (CAPRI), University of Antwerp, Universiteitsplein 1, 2610 Wilrijk, Belgium

29 Child and Adolescent Psychiatrist, General Childrens Hospital “Agia Sophia”, Athens, Greece

30 Department of Child and Adolescent Psychiatry, Public Pediatric Teaching Clinical Hospital, Medical University of Warsaw, 63A, Żwirki i Wigury Str., 02-091 Warsaw, Poland

31 Department of Child and Adolescent Psychiatry, Psychosomatics and Psychotherapy, University Hospital Essen, University of Duisburg-Essen, Duisburg, Germany 